Check for updates

Cite this: RSC Adv., 2017, 7, 30060

\title{
Plasma metabolic profiling and novel metabolite biomarkers for diagnosing prostate cancer $\dagger$
}

\begin{abstract}
Yunbo Zhao,,$^{a}$ Hongmei Lv, $t^{b}$ Shi Qiu, ${ }^{c}$ Lijuan Gaoc and Huazhang Ai (DD *c
Prostate cancer (PCa) is the second leading cause of cancer death among men and associated with profound metabolic changes. It is important to discover new biomarkers for the diagnosis of PCa and metabolomics technology has made progress toward identifying metabolic alterations in diseases that may provide clinically useful biomarkers. The purpose of this work was to determine the distinctive metabolic signatures from PCa. Plasma samples from PCa patients and age-matched healthy controls were investigated using high resolution ultrahigh performance liquid chromatography-mass spectrometry (UPLC/MS) and the datasets were analyzed using pattern recognition methods. Metabolic differences among PCa and control subjects were identified based on multivariate statistical analyses. As a result, 19 distinguishable metabolites were detected and 11 metabolic pathways were established. The altered metabolic pathways were associated with synthesis and degradation of ketone bodies, and phenylalanine metabolism, etc. To demonstrate the utility of plasma biomarkers for the diagnosis of PCa, three metabolites comprising sarcosine, acetylglycine and coreximine that contributed to the combined model were selected as candidate biomarkers. Distinctive signature with these metabolites could significantly increase the diagnostic performance of PCa. In this study, metabolomics technology has proved to be a powerful tool for the discovery of new biomarkers for disease and suggest that panels of metabolites may be valuable to translate our findings to clinically useful diagnostic tests.
\end{abstract}

Received 18th April 2017

Accepted 30th May 2017

DOI: $10.1039 / c 7 r a 04337 f$

rsc.li/rsc-advances

\section{Introduction}

Prostate cancer (PCa) is the second most diagnosed cancer in men, and principally affecting men over 50 years old worldwide. ${ }^{1}$ Its incidence and associated, mortality rates of PCa are increasing rapidly with the growing aging population. ${ }^{2}$ However, the diagnosis and possibility of predicting the outcome for PCa patients remain troublesome. Currently, detection of PCa mainly involves testing of serum prostatespecific antigen (PSA) level. ${ }^{3-5}$ However, recent studies suggested that PSA is not a specific biomarker of PCa and the diagnostic paradigm suffers from low specificity of PSA. There are further concerns regarding possible over-diagnosis of PCa by PSA in patients with limited potential for disease progression. ${ }^{6}$ Although several promising candidates have been

\footnotetext{
${ }^{a}$ Department of General Surgery, The First Affiliated Hospital of Jiamusi University, Jiamusi 154003, China

${ }^{b}$ Jiamusi College, Heilongjiang University of Chinese Medicine, Jiamusi 154007, China 'College of Pharmacy, Department of Rheumatology, First Affiliated Hospital, Heilongjiang University of Chinese Medicine, Heping Road 24, Xiangfang District, Harbin 150040, China. E-mail: aihuaz@yeah.net; Fax: +86-451-86053141; Tel: +86451-86053141

$\dagger$ Electronic supplementary information (ESI) available. See DOI: $10.1039 / \mathrm{c} 7 \mathrm{ra} 04337 \mathrm{f}$

\$ Co-authors.
}

proposed,,$^{7-9}$ the ideal biomarker or group of biomarkers for PCa remains elusive. Therefore, it is necessary to search a rapid and high efficiency method for increasing the accuracy of diagnosis.

Biomarkers can improve PCa diagnosis and treatment. There is a close relationship between metabolism and cancer. Metabolomics is a promising avenue for biomarker discovery. ${ }^{10}$ Recent reports suggest that molecular biomarkers have been identified and provided by metabolomic studies. ${ }^{11}$ Metabolomics is an approach that allows the analysis of the entire metabolites in a biological system. In metabolomics, a large number of small molecules (metabolites) can be detected in a single sample. It has been expanding in scope, from its primary use in the clinic for a new diagnostic role in diseases. ${ }^{12}$ Metabolomics could provide insights of the physiological status from a biological system. The alterations in the normal metabolome may be indicative of diseases. ${ }^{13-15}$ These alterations in the normal metabolome have the potential to discover new diagnostic biomarkers for the diseases. The diagnostic capacity of plasma metabolite analyses has also been confirmed by several studies. ${ }^{16}$

In this study, we mainly aim to identify the potential metabolic biomarkers and further to explore whether plasma metabolomics method could be used for diagnosing PCa. Metabolites were measured after analysis of plasma samples 
with ultrahigh performance liquid chromatography coupled to mass spectrometry (UPLC-MS) instrumentation. Multivariate parametric statistical test and receiver operating characteristics analysis was performed to evaluate diagnostic performance of the metabolites. Besides, we will significantly contribute to a deeper understanding of the biochemical metabolic networks and pathways in PCa.

\section{Experimental}

\section{Chemicals and reagents}

Methanol (HPLC grade) and acetonitrile (HPLC grade) were purchased from Merck (Darmstodt, Germany). Ultrapure water was provided by a Milli-Q water purification system (Millipore, Billerica, USA). Formic acid and leucine enkephalin was purchased from Sigma-Aldrich (St. Louis, MO, USA).

\section{Study participants}

All patients and control individuals consisted of healthy men were recruited from the First Affiliated Hospital, Heilongjiang University of Chinese Medicine, and all patients signed informed consents before the study began. Criteria for selection of the PCa group was the following: PCa diagnosis based on digital rectal examination, transrectal ultrasonography and examination of biopsy tissue sample, no other coexisting cancers, no PCa treatment. The control group has no cancer and no chronic diseases. A total of 32 patients together with 32 normal control cases were enrolled. Characteristics of the PCa group and control group were reflected in ESI Table $1 . \dagger$ Before collection of the samples, patients had given consent and had undergone therapeutic interventions. The study was endorsed by the Review Board of Heilongjiang University of Chinese Medicine (HUCM-2016-0652) and complied with the provisions of the Good Clinical Practice Guidelines and the Declaration of Helsinki.

\section{Sample collection and preparation for metabolomics analysis}

Plasma samples were collected from PCa patients and control group. Fasting venous blood samples were collected using vacuum blood collection tube that contained anticoagulant dipotassium EDTA. Plasma was separated by centrifugation at $5000 \mathrm{rpm}$ for $10 \mathrm{~min}$ and the supernatant was stored at $-80{ }^{\circ} \mathrm{C}$ until further analysis. To ensure the stability and repeatability of UPLC/MS, the blank samples and quality control samples were used in this study. All the plasma samples were thawed at $4{ }^{\circ} \mathrm{C}$ and a volume $300 \mu \mathrm{L}$ of cold methanol were added to 100 $\mu \mathrm{L}$ of plasma for deproteinization, and then centrifuged at $5000 \mathrm{rpm}$ for $10 \mathrm{~min}$. Next, the supernatants were recovered, evaporated using a vacuum rotary dryer and re-suspended in $100 \mu \mathrm{L}$ acetonitrile/water $(1: 3, \mathrm{v} / \mathrm{v})$, vortex-mixed for $5 \mathrm{~min}$, then centrifuged at $5000 \mathrm{rpm}$ for $10 \mathrm{~min}$, and the supernatant was held for UPLC/MS analysis.

\section{LC-MS analysis}

Chromatographic separation was performed on Ultra Performance Liquid Chromatography (UPLC, Waters Corporation, Manchester, UK) system. The instrument was equipped with a degasser, binary pump, thermostatted

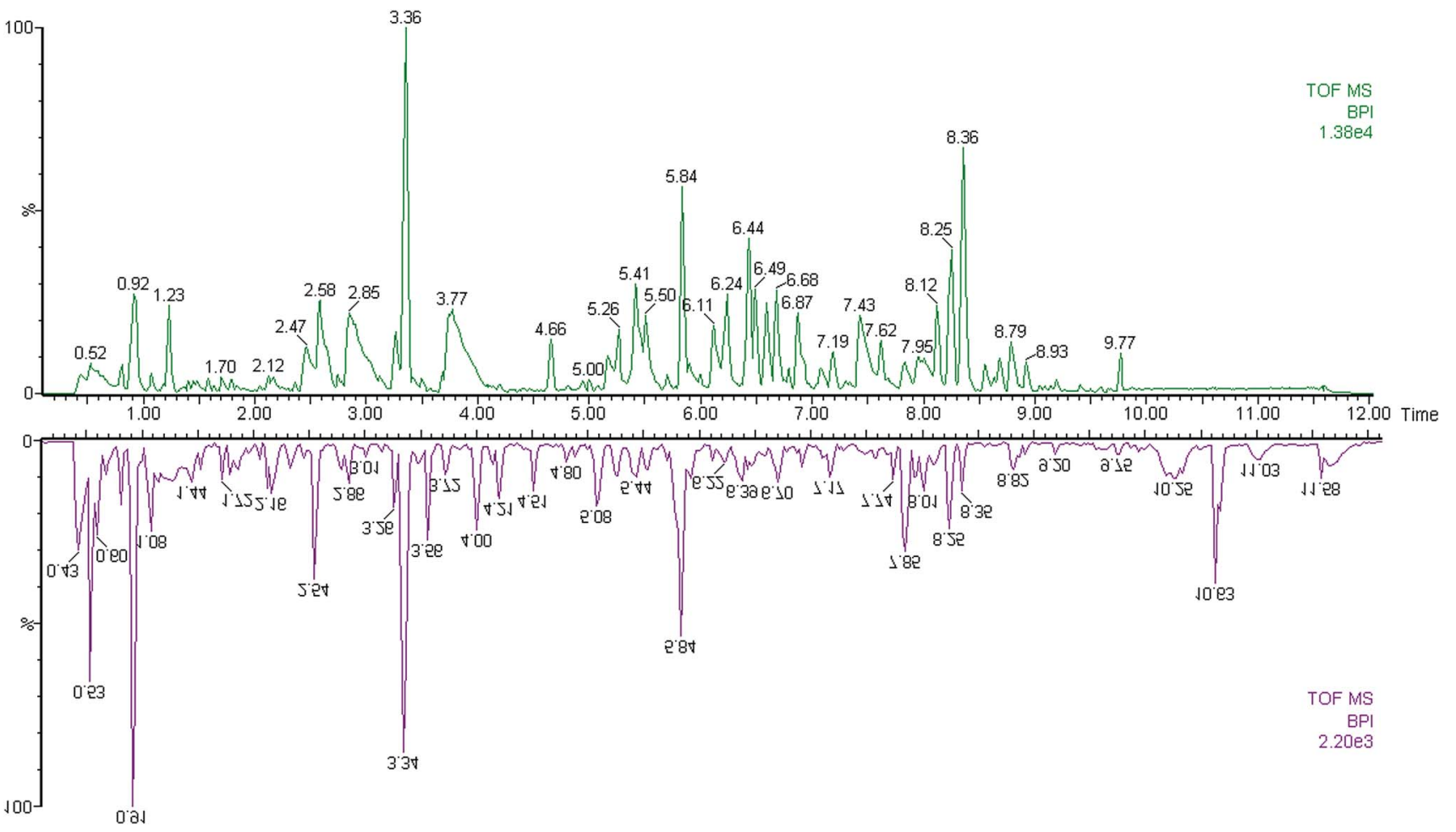

Fig. 1 Typical base peak chromatograms of plasma samples from control subjects (up) and PCa patients (down) obtained by UPLC-MS tool. 
autosampler, and column oven (Waters, Milford, USA). Samples were performed on UPLC ${ }^{\text {TM }}$ BEH C18 column (50 $\mathrm{mm} \times 2.1 \mathrm{~mm}$ i.d., $1.7 \mu \mathrm{m}$, Waters, Milford, USA). The column was maintained at $45{ }^{\circ} \mathrm{C}$. The flow rate throughout the separation was maintained at $0.5 \mathrm{~mL} \mathrm{~min}^{-1}$. The mobile phase was consisted of acetonitrile/water (50:50 v/v) containing $0.1 \%$ formic acid (solvent $\mathrm{A}$ ) and acetonitrile/water (90:10 v/v) containing $0.1 \%$ formic acid (solvent B). The gradient conditions were $0-2 \min 1-15 \%$ B; $2-6 \min 15-50 \%$ $\mathrm{B} ; 6-10 \mathrm{~min} 50-95 \% \mathrm{~B} ; 10-12 \mathrm{~min} 95 \% \mathrm{~B}$ followed by reequilibration to the initial starting condition. The samples were kept at $4{ }^{\circ} \mathrm{C}$, and a constant volume of $4 \mu \mathrm{L}$ was injected for analysis.

MS full scan was performed positive ion mode and negative ion mode using a time of flight mass spectrometer (Waters, Milford, USA), which was coupled to the UPLC system. The mass
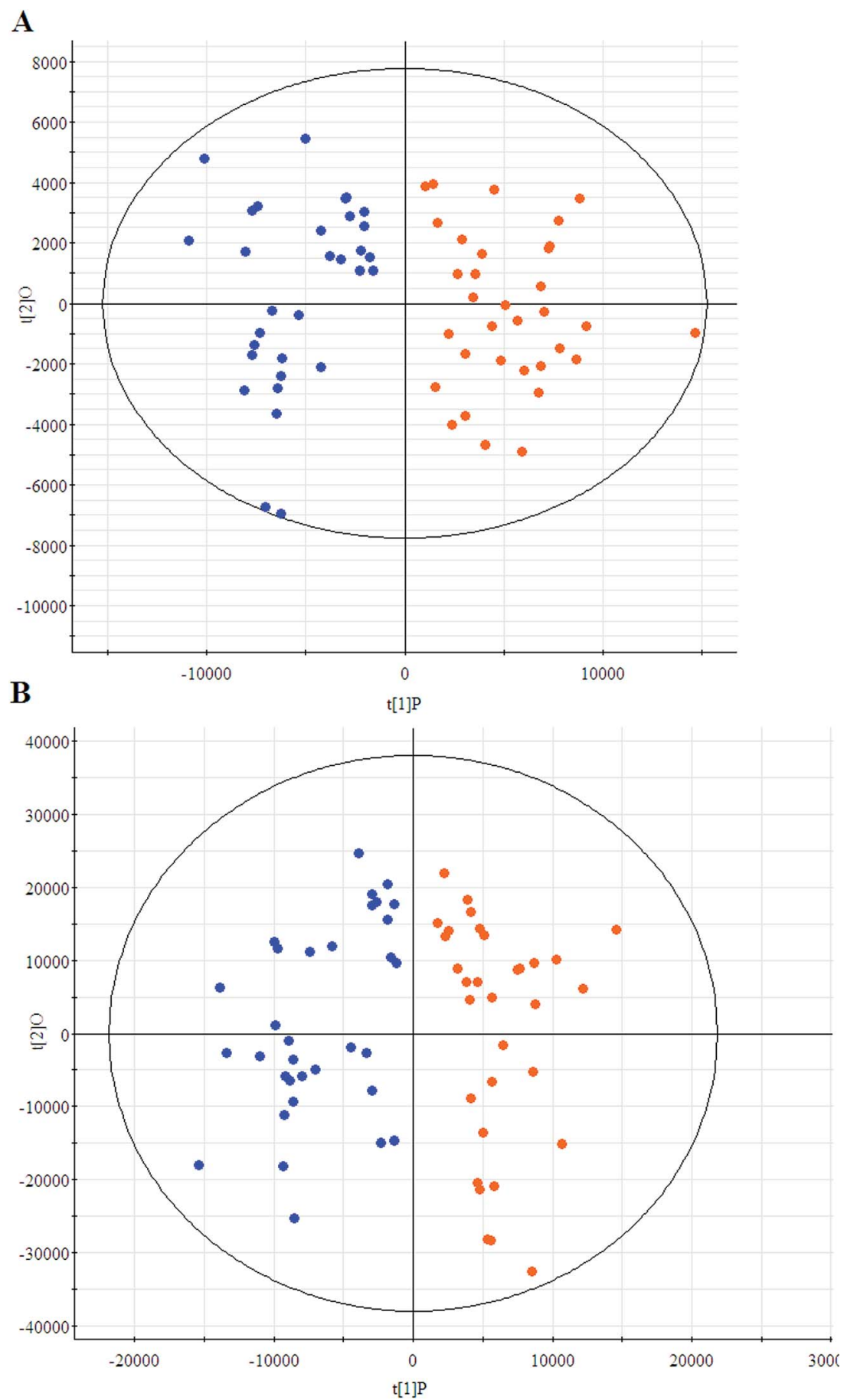

Fig. 2 Principal component analysis score plots of the patients (blue) and controls (red) group in positive ion model (A) and negative ion model (B). 
scanning range was $50-1500 \mathrm{~m} / \mathrm{z}$ in the full scan mode, with a capillary voltage of $3000 \mathrm{~V}$, a collision gas flow rate of $10 \mathrm{~L}$ $\mathrm{min}^{-1}$, and a nebulizer gas flow rate of $35 \mathrm{~L} \mathrm{~min}^{-1}$, the capillary temperature of $300{ }^{\circ} \mathrm{C}$, the source temperature of $120{ }^{\circ} \mathrm{C}$, and desolvation gas temperature of $400^{\circ} \mathrm{C}$. Nitrogen was used as the collision gas at a collision cell pressure of $2.0 \times 10^{-5}$ Torr. The flow rates of cone and desolvation gas were set at $60 \mathrm{~L} \mathrm{~h}^{-1}$ and $400 \mathrm{~L} \mathrm{~h}^{-1}$, respectively.

\section{Multivariate statistical analyses}

LC-MS data were analyzed to identify potential discriminant biomarkers. The peak finding, filtering, and alignment were performed by MarkerLynx 4.1 application manager (Waters, Manchester, UK). The MS matrix was then introduced to EZinfo 2.0 software (Waters, Manchester, UK) for orthogonal signal correction-partial least squares discriminant analysis (OPLSDA) which produces a score plot showing the separation of the groups based on the content of the loading discriminatory metabolites. $R^{2} Y$ (cum) and $Q^{2}$ (cum) parameters were used for the evaluation of the models, indicating the fitness and prediction ability, respectively. VIP-plot from OPLS-DA was utilized to rank the metabolites, according to their importance to the classification. The differential metabolites were identified based on the MS/MS fragment comparison with the standard compounds, or via search of the candidate compounds in the database including the HMDB, METLIN and Chemspider.

\section{Metabolic pathway and network analysis}

To identify the most relevant metabolic pathways involved in PCa, metabolic pathway analysis was employed to perform the pathway topology analysis using the software MetaboAnalyst 3.0 (http:/www.metaboanalyst.ca). The metabolites with VIP scores $>1.0$ in the partial least squares were examined and selected for their metabolic pathway analysis.

\section{Discrimination performance of potential biomarkers}

Metabolomics was performed to explore whether metabolomic signatures had the potential discrimination ability between PCa and control group. To obtain a final diagnostic score, receiver operating characteristic (ROC) curves were generated using the rocplot function program. It allows characterization of diagnostic accuracy, and evaluates the predictive accuracy. ROC curves were used to estimate the accuracy of the combined signatures model.

\section{Statistical analysis}

The areas under curve (AUC) of ROC curves were used to determine the diagnostic effectiveness of important metabolites using the online software MetaboAnalyst 3.0 (http:// www.metaboanalyst.ca). The SVM algorithm was used to calculate the importance of all variables and rank them. The $t$ test analysis of covariance was performed using SPSS software (version 19.0; SPSS, Inc., Chicago, IL), with $p<0.05$ deemed to be significant.

\section{Results}

\section{Baseline clinical characteristics}

Characteristics of the study population are presented in ESI Table $1 . \dagger$ The mean ages, sex, body mass index, 2 hour postprandial glucose, total cholesterol, triglycerides, systolic blood pressure, and diastolic blood pressure were not significantly different between patients with PCa cases and age-matched
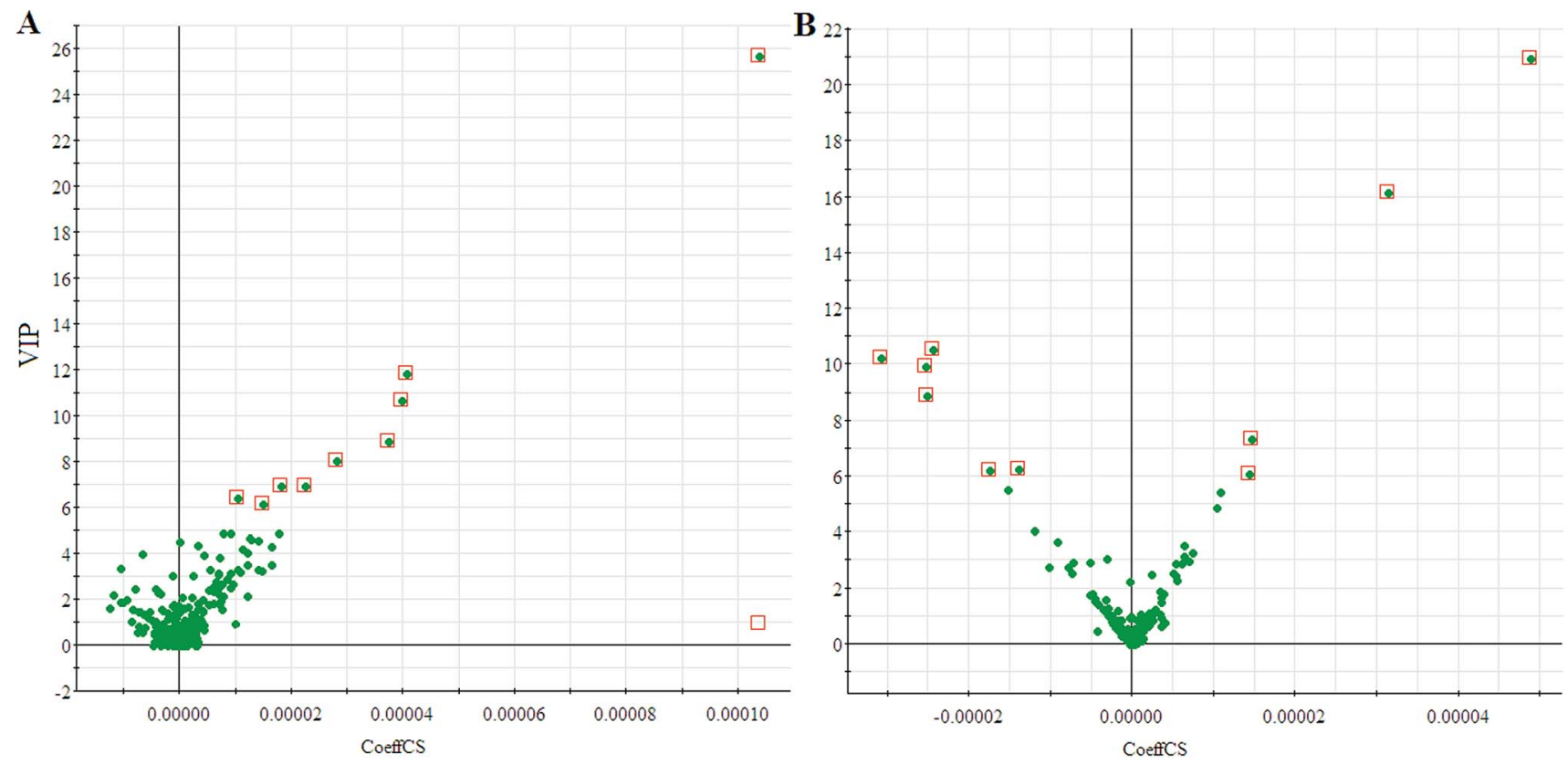

Fig. 3 VIP-plot for screening of significantly changed metabolites in positive electrospray mode (A) and negative electrospray modes (B). 


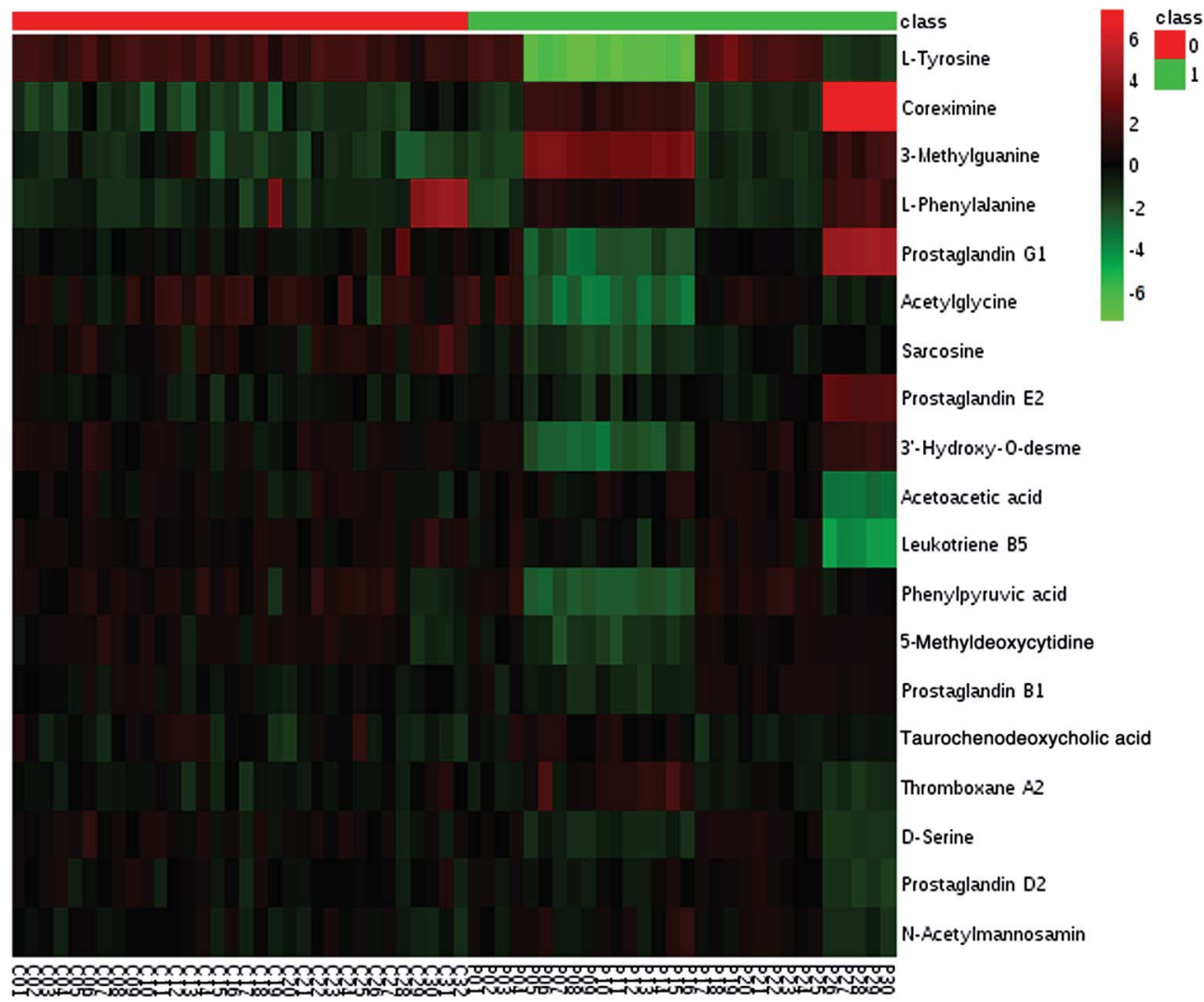

Fig. 4 Heatmap visualization analysis of metabolites statistically significant with a potential identity.

healthy controls. The PSA was $9.8 \pm 5.6$ for PCa patients, indicating a fairly advanced stage of this disease. The serum PSA level significantly differed between the controls and PCa patients $(P<0.05)$.

\section{Overall metabolomics analysis of plasma samples}

The first aim of this work was to analyze the global metabolic differences between PCa and health cases. To do this, we applied global metabolomics focusing on the profiles of low molecular weight metabolites. In this study, the plasma samples were analyzed by UPLC-MS tool. The separation conditions of plasma samples were optimized. Representative base peak intensity chromatograms of plasma samples from the controls and the PCa cases in positive ion mode were presented in Fig. 1. The serum spectra contained highintensity signals from the small molecules and the utilization of multivariate statistical approach could enlarge metabolite identification. The variables were exported into EZinfo 2.0 software (Waters, Manchester, UK) for multivariate data analysis to detect metabolite. Statistical evaluation by principal component analysis (PCA) showed a clearly different distribution in the positive ion model (Fig. 2A) and negative ion mode (Fig. 2B). The wider spread of the samples in the PCA was caused by altered levels of metabolites. OPLS-DA was then applied to evaluate the more marked variations and statistical meaning of those ions.

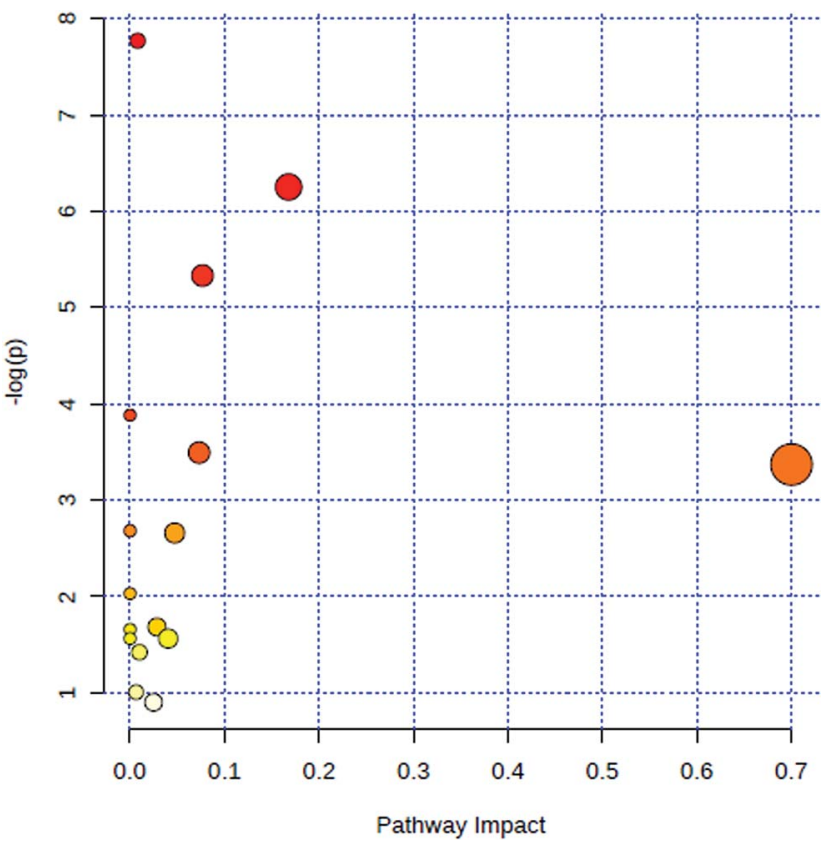

Fig. 5 Summary of metabolic pathways of significantly changed metabolites with MetPA. Circles represent metabolism (ESI Table S3†). All matched pathways are plotted according to $p$-value from pathway enrichment analysis and pathway impact score from pathway topology analysis. Colour gradient and circle size indicate the significance of the pathway ranked by $p$-value (yellow: higher $p$-values and red: lower $p$-values) and pathway impact score (the larger the circle the higher the impact score), respectively. 


\section{Plasma metabolites related to $\mathrm{PCa}$}

We employed an OPLS-DA statistical way termed VIP-plot to select metabolites that highly contributed to the behaviors. VIP-plot of PCa patients vs. control individuals was shown in Fig. 3, which is a scatter plot that combines the covariance and correlation. Higher values of VIP indicate metabolites that are more important to the classification. Variables with VIP value greater than 6 were considered as great value. A $t$ test was performed in variables with significant differences between PCa cases and control individuals $(P<0.01)$ were retained. According to the VIP scores, a total of 19 discriminate variables (listed in ESI Table $2 \dagger$ ) as interesting candidates were found in PCa. Elemental composition was estimated using the Masslynx 4.1 software. And then, it was finally confirmed by comparison with a standard sample. Then, the metabolites were analyzed using a heat map and it indicated that the two groups could be separated based on these metabolites (Fig. 4).

\section{Metabolite pathways related to $\mathrm{PCa}$}

All of the attributed metabolites were analyzed utilizing Metaboanalyst 3.0. The pathway analysis results showed the metabolic network reprogramming of PCa with detailed impact (Fig. 5). The most influenced metabolic pathway was set as a pathway influence $>0.01$. The biological pathway analysis revealed 11 metabolic pathways, including synthesis and degradation of ketone bodies, phenylalanine metabolism, arachidonic acid metabolism, glycine, serine and threonine metabolism, tyrosine metabolism, butanoate metabolism, propanoate metabolism, amino sugar and nucleotide sugar metabolism, primary bile acid biosynthesis, phenylalanine, tyrosine and tryptophan biosynthesis, arginine and proline metabolism (ESI Table $3 \dagger$ ). To visualize biological connectivity of the significantly changed metabolites, the networkgenerating algorithm of ingenuity pathway analysis was used to maximize the interconnectedness of molecules based on the database. It revealed that the PCa patients possessed a highly unique metabolic phenotype characterized by these metabolic pathways (Fig. 6).

\section{Potential diagnostics of metabolites}

We have performed ROC analysis to further characterize the predictive value of these individual metabolites independently. We found 4 metabolites (sarcosine, acetylglycine, coreximine

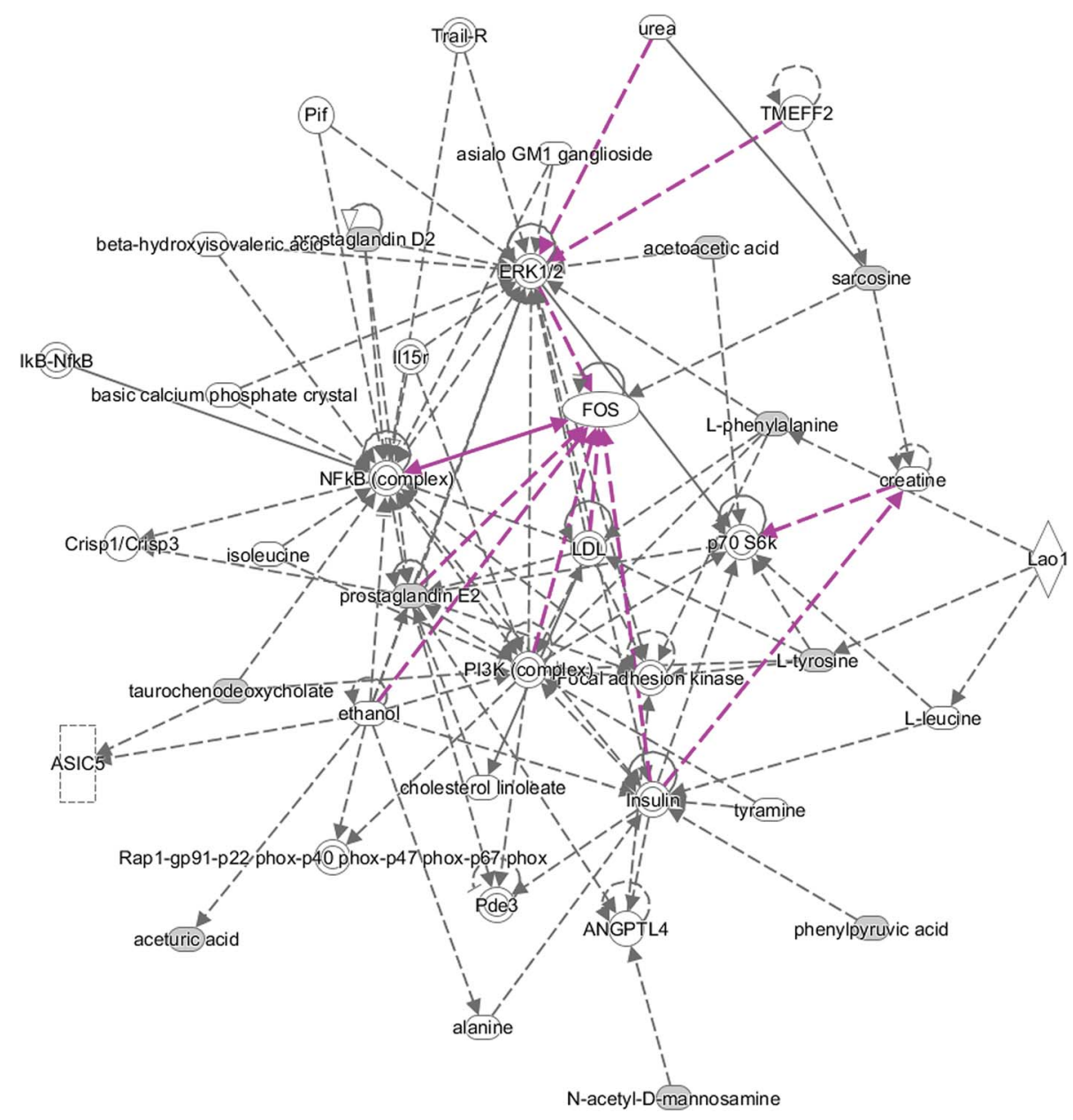

Fig. 6 Receiver operator characteristic analysis of metabolites significantly associated with PCa. 
Sarcosine

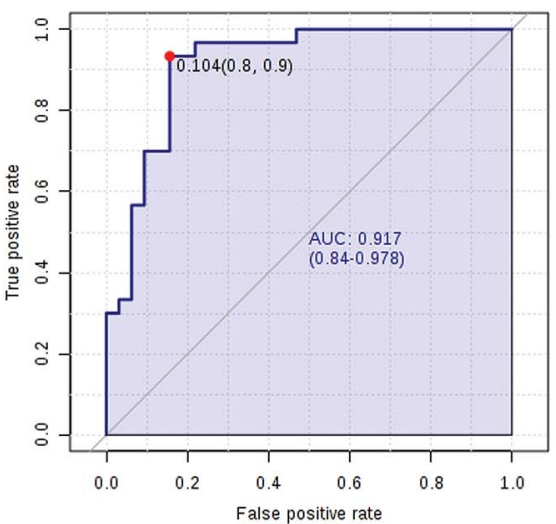

3-Methylguanine

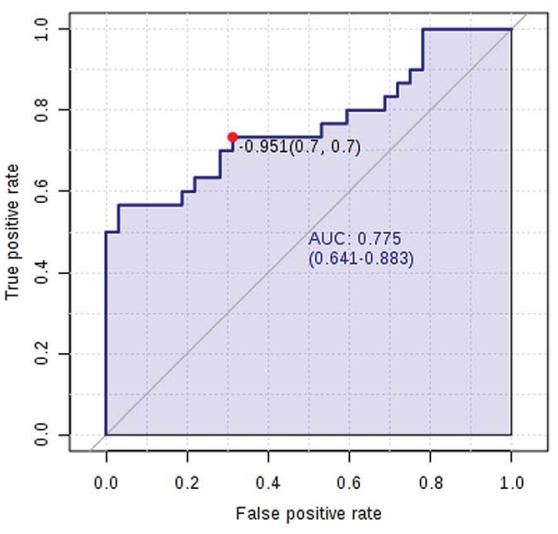

Acetylglycine

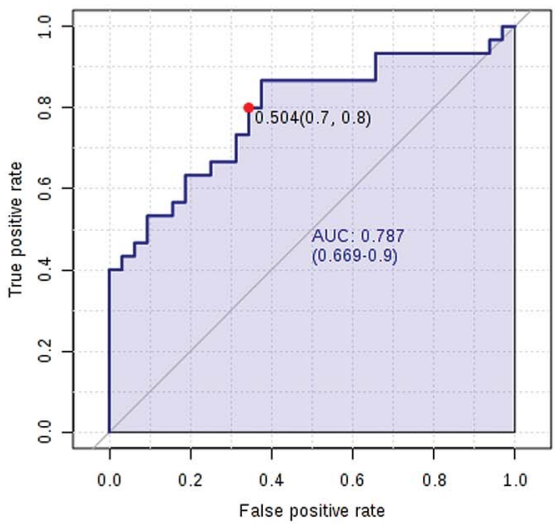

5-Methyldeoxycytidine

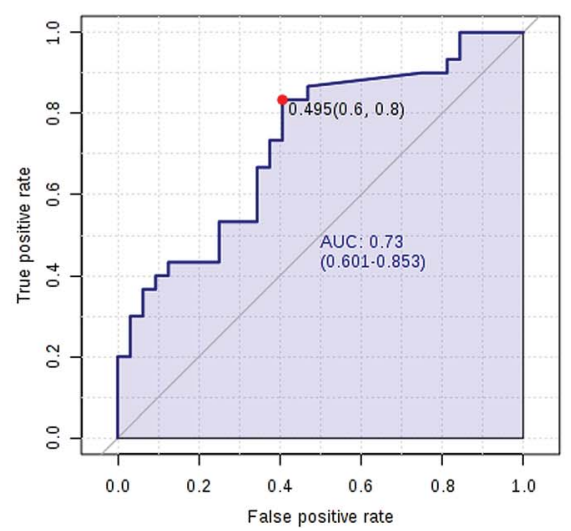

Coreximine

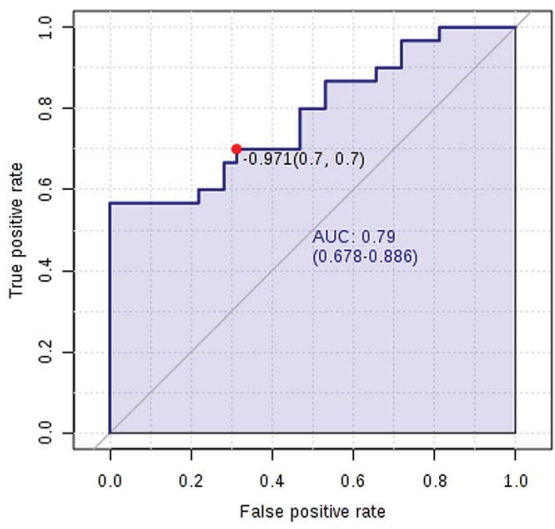

D.Serine

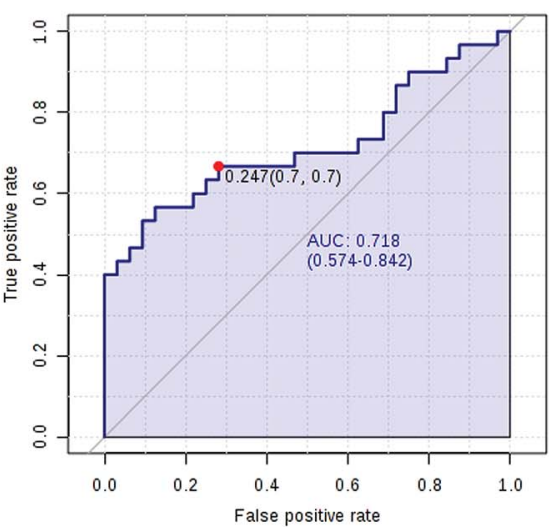

Fig. 7 Ingenuity pathway analysis of the significantly changed metabolites.

and 3-methylguanine $(p=0.0000))$ with an area under the curve (AUC) >0.7. Among the metabolites, we found with highest significant the sarcosine (Fig. 7 and ESI Table $4 \dagger$ ). The results

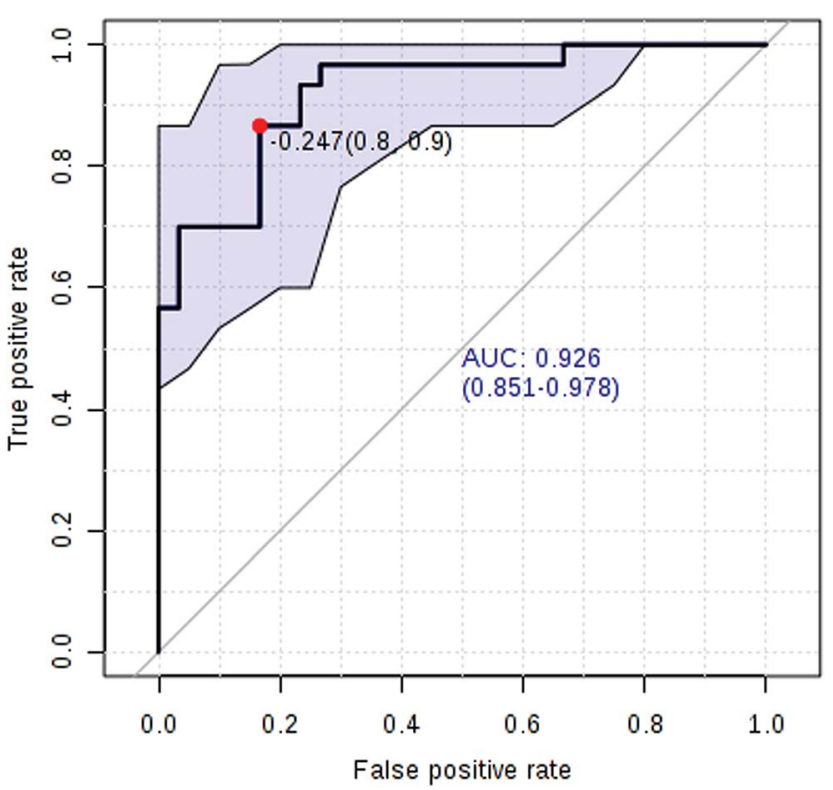

Fig. 8 ROC curve analysis for the serum prostate-specific antigen level. of univariate ROC curve analyses indicated that in the plasma samples, 7 metabolites with high AUC above 0.70 were: sarcosine, acetylglycine, coreximine, 3-methylguanine, 5-methyldeoxycytidine, D-serine, and leukotriene B5 (ESI Table $4 \dagger$ ).

\section{PCa diagnostics related metabolites}

Currently, detection of PCa using test of PSA level showed a low discriminator (AUC $=92.6 \%$ ) (Fig. 8). It is necessary to establish a novel method for increase the accuracy of diagnosis. To improve the prediction of $\mathrm{PCa}$, a combination of more than one discriminatory metabolite was developed via logistic regression analysis (Fig. 9A). Fig. 9A shows that the combination of metabolites was a better discriminator (AUC $>94 \%$ ) than each metabolite individually (AUC $<94 \%$ ), which reinforced the improved capacity of biomarker patterns to make a distinction between different groups. Thus, the distinctive signature with the 3 metabolites had achieved the highest AUC value and significantly increased the diagnostic performance of PCa. The designed model performance was assessed by the AUC. Notably, for the feature ranking method, top 3 metabolites including sarcosine, acetylglycine and coreximine contributed to the combined model (Fig. 9B). Thus, the distinctive signature of the 3 metabolites significantly increased the diagnostic performance of PCa. 
A

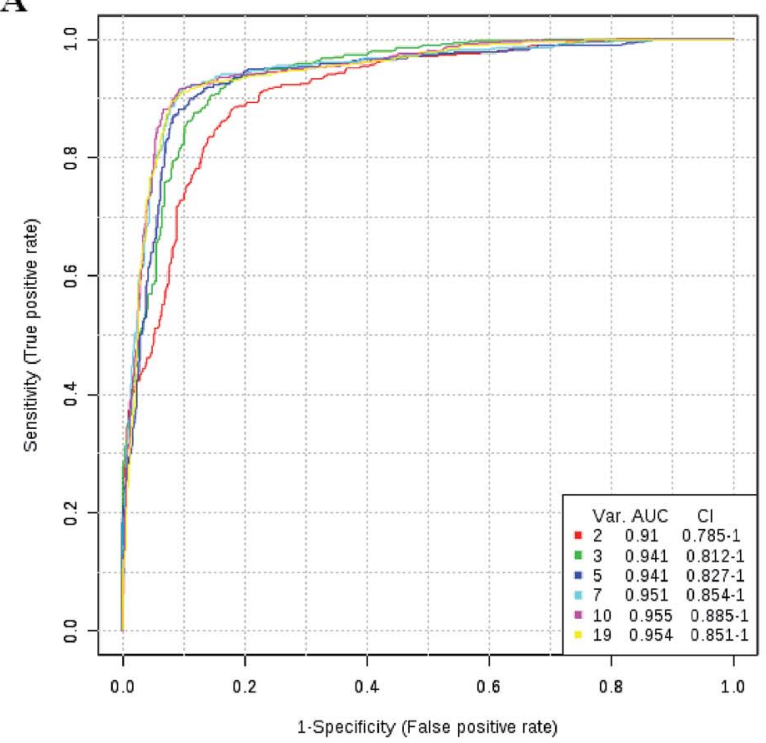

B

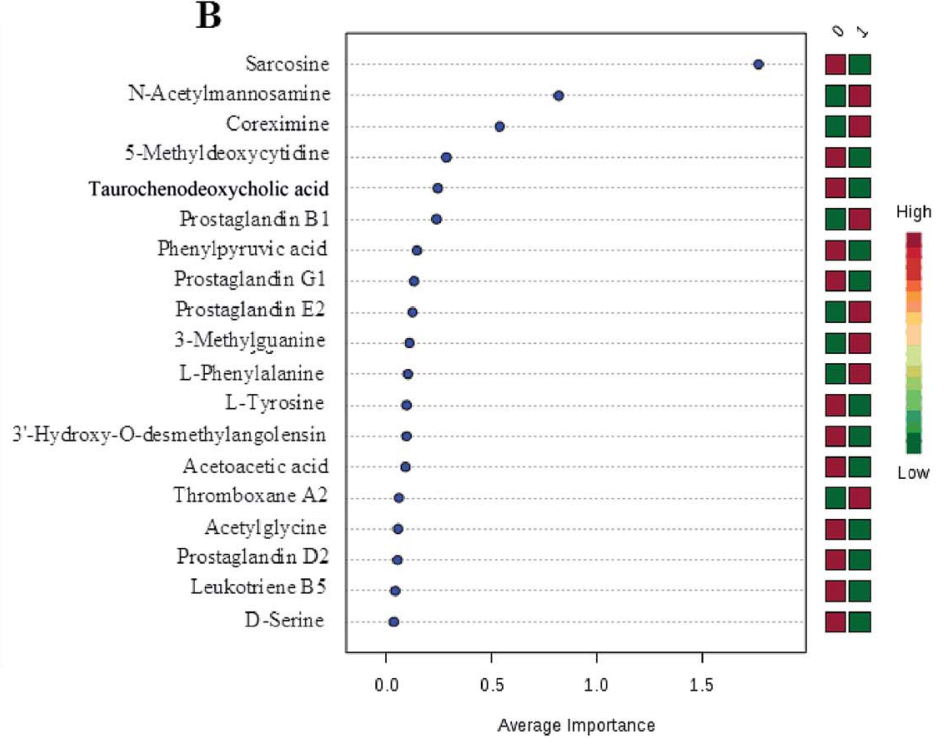

Fig. 9 ROC curve for the predictive model. A combination metabolites model calculated from the logistic regression analysis (A); features ranked by their contributions to classification accuracy (B).

\section{Discussion}

PCa is a significant cause of morbidity and mortality among men worldwide. Current diagnosis of PCa is based on prostate biopsies, but it can lead to false-negative and false-positive results. ${ }^{17}$ The better biomarkers are urgently needed to improve the specificity of PCa diagnosis and avoid unnecessary biopsies. For the discovery of new biomarkers, the application of omics is emerging, especially metabolomics screening techniques. Cancer has been associated with marked metabolic change. Metabolites can be altered in cancer, because the metabolites produced are indicators of what is happening in the metabolism of disease conditions. ${ }^{18}$ Metabolomics has significantly contributed to a deeper understanding of the metabolic pathways in cancer. Some studies have demonstrated that dysregulated metabolism is associated with the survival of cancers in patients. ${ }^{19}$ Identification of the dysfunctional metabolic pathways of cancer via metabolomics can be used to discover biomarkers and targets. ${ }^{20}$ Some previous studies have attempted to use metabolomics to discover biomarkers that might apply the detection of cancer. ${ }^{21}$ Plasma is a readily available biofluid that may contain metabolites of interest for diagnosis of diseases. Indeed, a growing number of studies have used plasma-based metabolomics as a method of discovering biomarkers for diagnosing $\mathrm{PCa}^{22}$

In the present study, a non-targeted UPLC/MS plasma metabolism method was used to explore the metabolic characteristics related to the PCa patients and to screen meaningful predictors. We compared plasma metabolic profiles of 32 patients with PCa to identify its metabolic signatures. PCA showed a clearly different distribution in the positive ion mode and negative ion mode. The wider spread of the samples in the PCA was caused by different levels of metabolites. We employed VIP-plot of OPLS-DA statistical approach to select metabolites that highly contributed to the clear separation behaviors. According to the VIP scores, a total of 19 discriminate variables as interesting candidates were found in PCa. We identified 9 metabolites in $\mathrm{ESI}^{+}$mode and 10 metabolites in $\mathrm{ESI}^{-}$mode related to the PCa (Fig. 3 and ESI Table $2 \dagger$ ). In the present study, we provided evidence that there exists a high relationship between these metabolites and PCa. The most influenced metabolic pathway includes synthesis and degradation of ketone bodies, phenylalanine metabolism, arachidonic acid metabolism, glycine, serine and threonine metabolism, tyrosine metabolism, butanoate metabolism, propanoate metabolism, amino sugar and nucleotide sugar metabolism, primary bile acid biosynthesis, phenylalanine, tyrosine and tryptophan biosynthesis, arginine and proline metabolism.

In recent years, numerous findings have been published that using the marker candidates could potentially improve the diagnosis and help to identify patients. ${ }^{23-26}$ Establishing a diagnostic model to predict the PCa was difficult due to the distinct metabolic profile of PCa consisting of the 11 altered metabolic pathways and 19 corresponding metabolites. Now, detection of PCa using test of PSA level showed a low discriminator (AUC = 92.6\%). We then performed ROC analysis to characterize the predictive value of those metabolites for discriminating PCa. Evaluation of biomarkers by ROC analysis showed that 7 metabolites with high AUC above 0.70. A combination of more than one discriminatory metabolite was developed via logistic regression analysis to construct an effective diagnostic model for PCa. Notably, for the feature ranking method, we found that top 3 metabolites including sarcosine, acetylglycine and coreximine contributed to the combined model and had achieved the highest AUC value (AUC $=94.1 \%$ ). Therefore, the distinctive signature of the 3 metabolites significantly increased the diagnostic performance of PCa. It revealed the potential 
pathogenesis of $\mathrm{PCa}$, and also provided a feasible diagnostic tool for PCa populations through detection of plasma metabolites.

The development of biomarkers to diagnose PCa is meaningful for both patient care and research. In this study, we had investigated whether the alterations in plasma metabolites are available for detection of $\mathrm{PCa}$, and performed based on the UPLC/MS technique supported by the advanced chemometric analysis. The aim of this study is to find metabolite biomarkers that would allow the discovery and diagnosis of PCa. We found that the combination of metabolites was a better discriminator than each metabolite individually. In this study, plasma metabonomics analysis for identifying potential biomarkers to diagnose PCa was successfully demonstrated, which has the advantages of reliable, simple, and low-cost. In conclusion, our research highlights the potential advantages of the plasma metabolomics in real clinical diagnostics.

\section{Conclusions}

This is the first study to discover the ideal biomarker, or set of biomarkers, significantly increase the diagnostic performance of PCa. In this study, we applied plasma metabolomics approach based UPLC-MS combined with pattern recognition approach in both positive and negative modes to investigate the discriminated variables. 17 potential biomarkers in the PCa subjects were significantly different from the control subjects. Additionally, plasma metabolomics study presented the dysregulated metabolism related to the PCa and several altered pathways aberrantly expressed were found at metabolic level, including synthesis and degradation of ketone bodies, phenylalanine metabolism, etc. We found that top 3 metabolites including sarcosine, acetylglycine, and coreximine were selected as candidate biomarkers and contributed to the combined model as well as achieved the highest diagnosis value of PCa. These findings provide the basis for a novel diagnostic metabolomics paradigm that could improve the clinical diagnostics of PCa patients. Furthermore, future studies are needed to validate the findings in larger number of patients and investigate their potential clinical utilities in PCa.

\section{Conflict of interest}

The authors declare no competing financial interests.

\section{Acknowledgements}

This work was supported by grants from the Key Program of Natural Science Foundation of State (Grant No. 81302905), Natural Science Foundation of Heilongjiang Province of China (H2015038), Doctoral Science Foundation of Heilongjiang University of Chinese Medicine (2014bs08), Youth Innovative Talent Program of Heilongjiang Province of China (UNPYSCT2015118).

\section{References}

1 O. Twu, D. Dessí, A. Vu, F. Mercer, G. C. Stevens, N. De Miguel and P. J. Johnson, Proc. Natl. Acad. Sci. U. S. A., 2014, 111, 8179-8184.

2 L. P. Bokhorst, C. H. Bangma, G. J. van Leenders, J. J. Lous, S. M. Moss, F. H. Schröder and M. J. Roobol, Eur. Urol., 2014, 65, 329-336.

3 J. C. Byrne, M. R. Downes, N. O'Donoghue, C. O'Keane, A. O'Neill, Y. Fan and R. W. G. Watson, J. Proteome Res., 2008, 8, 942-957.

4 A. Heidenreich, P. A. Abrahamsson, W. Artibani, J. Catto, F. Montorsi, H. Van Poppel and N. Mottet, Eur. Urol., 2013, 64, 347-354.

5 S. H. Strand, T. F. Orntoft and K. D. Sorensen, Int. J. Mol. Sci., 2014, 15, 16544-16576.

6 M. S. Leapman and P. R. Carroll, Urol. Oncol., 2017, 35, 4250.

7 V. Medina-Villaamil, S. Martinez-Breijo, P. Portela-Pereira, M. Quindos-Varela, I. Santamarina-Cainzos, L. M. AntonAparicio and F. Gómez-Veiga, Actas Urológicas Españolas, 2014, 38, 633-639.

8 V. M. Barnes, A. D. Kennedy, F. Panagakos, W. Devizio, H. M. Trivedi, T. Jönsson and F. A. Scannapieco, PloS one, 2014, 9, e105181.

9 J. Zheng, R. A. Dixon and L. Li, Anal. Chem., 2012, 84, 1080210811.

10 Q. Liang, H. Liu, H. Xing, Y. Jiang and A. H. Zhang, RSC Adv., 2016, 6, 43293-43298.

11 Q. Liang, H. Liu, T. Zhang, Y. Jiang, H. Xing and A. H. Zhang, RSC Adv., 2015, 5, 102204-102209.

12 T. J. Wang, M. G. Larson, R. S. Vasan, S. Cheng, E. P. Rhee, E. McCabe and C. J. O'donnell, Nat. Med., 2011, 17, 448-453.

13 Q. Liang, H. Liu, Y. Jiang, H. Xing, T. Zhang and Z. Ai-hua, RSC Adv., 2016, 6, 11008-11013.

14 Q. Liang, H. Liu, X. Li and A. H. Zhang, RSC Adv., 2016, 6, 75499-75504.

15 A. H. Zhang, H. Sun, Y. Han, G. L. Yan, Y. Yuan, G. C. Song and X. J. Wang, Anal. Chem., 2013, 85, 7606-7612.

16 X. Zang, C. M. Jones, T. Q. Long, M. E. Monge, M. Zhou, L. D. Walker and F. M. Fernandez, J. Proteome Res., 2014, 13, 3444-3454.

17 W. Struck-Lewicka, M. Kordalewska, R. Bujak, A. Y. Mpanga, M. Markuszewski, J. Jacyna and M. J. Markuszewski, J. Pharm. Biomed. Anal., 2015, 111, 351-361.

18 D. Kumar, A. Gupta, A. Mandhani and S. N. Sankhwar, J. Proteome Res., 2015, 14, 1455-1464.

19 A. M. Mondul, S. C. Moore, S. J. Weinstein, S. Männistö, J. N. Sampson and D. Albanes, Metabolomics, 2014, 10, 5.

20 A. Zhang, G. Yan, Y. Han and X. Wang, Appl. Biochem. Biotechnol., 2014, 174, 6-12.

21 Y. Zhang, P. Liu, Y. Li and A. H. Zhang, RSC Adv., 2017, 7, 6780-6787.

22 Q. Liang, H. Liu, L. X. Xie, X. Li and A. H. Zhang, RSC Adv., 2017, 7, 2587-2593. 
23 A. Zhang, H. Sun and X. Wang, RSC Adv., 2017, 7, 1496614973.

24 Y. Li, S. Qiu and A. H. Zhang, Anal. Methods, 2016, 8, 32843290.
25 X. Wang, J. Li and A. H. Zhang, $R S C A d v ., 2016$, 6, 6304963057.

26 Y. Zhou, R. Song, C. Ma, L. Zhou, X. Liu, P. Yin and G. Xu, Oncotarget, 2017, 8, 20719. 